\title{
Antitachycardia Pacemakers in Congenital Heart Disease
}

\author{
Anna N. Kamp, MD, MPH, ${ }^{*}$ Martin J. LaPage, MD, MS, ${ }^{\dagger}$ Gerald A. Serwer, MD, ${ }^{\dagger}$ \\ Macdonald Dick II, MD, ${ }^{\dagger}$ and David J. Bradley, $\mathrm{MD}^{\dagger}$ \\ ${ }^{*}$ Cardiology, The Heart Center, Nationwide Children's Hospital, Ohio State University, Columbus, Ohio, USA; ${ }^{\dagger}$ Pediatric \\ Cardiology, University of Michigan, Ann Arbor, Mich, USA
}

\section{A B S T R A C T}

Background. Many patients with congenital heart disease (CHD) acquire rhythm abnormalities related to their repair, most commonly intraatrial reentrant tachycardia (IART). Treatment of IART in CHD is often multifaceted, and may include medication, ablation, and pacing. Evidence regarding the use of antitachycardia pacing therapies is limited.

Objective. The aim of the study is to define the use and efficacy of antitachycardia pacing in patients with CHD at a single center.

Results. Eighty implants were performed on 72 patients between 2000 and 2010. Follow-up data of more than 3 months were available for 56 patients; median follow-up time was 2.8 years. Twenty (36\%) patients received successful antitachycardia pacing at a median 1.3 years postimplant. For those patients with IART after implant, antitachycardia pacing was successful in $57 \%$. Patients with two-ventricle repairs were more likely to have successful antitachycardia pacing than those with one-ventricle palliation $(45 \%$ vs. $17 \%, P=.04)$. Patients with documented IART had more successful antitachycardia pacing than those with no documented atrial tachycardia prior to implant ( $46 \%$ vs. $7 \%, P=.006)$. Early complications of antitachycardia pacemaker implant occurred in six patients $(11 \%)$; late complications after implant occurred in three patients (5.6\%). Of the initial 72 patients implanted, there were six deaths (8\%).

Conclusions. Antitachycardia pacing therapies were successful in the majority of CHD patients who had IART after implant. Patients without documented atrial tachycardia prior to implant were unlikely to require or receive successful therapy from antitachycardia pacemaker. Those patients postatrial switch procedure who had documented IART prior to implant had the highest incidence of successful antitachycardia pacing therapies. Antitachycardia pacemaker implantation is an adjunct to the management of IART in CHD patients, but may not benefit patients who have not yet demonstrated IART.

Key Words. Congenital Heart Disease; Atrial Tachycardia; Intraatrial Reentrant Tachycardia; Antitachycardia Pacemakers

\section{Introduction}

$\mathrm{M}$ any patients with congenital heart disease (CHD) acquire rhythm abnormalities related to their repairs, including atrial tachycardias (ATs), sinus node dysfunction (SND), or complete heart block (CHB). Intraatrial reentrant tachycardia (IART) is the most common tachycardia mechanism in adults with CHD, particularly those with complex CHD. ${ }^{1}$ For those with bradycardia pacing indications and IART, there is often

Congenit Heart Dis. 2015;10:180-184 a multifaceted approach to rhythm management, including medications, ablation, and antitachycardia pacing (ATP)..$^{2-4}$

An antitachycardia pacemaker (ATPM) may be effective for patients with recurrent IART refractory to other medical management. Data from a multicenter study of an earlier ATPM device show ATP to be effective in terminating atrial arrhythmias in over half of identified AT episodes without adverse effects. ${ }^{5}$ More recently, data on a newer model ATPM demonstrated successful termina- 
tion of IART in almost half of all episodes, and suggested that atrial ATP was safe in CHD. ${ }^{6}$

Limited evidence exists regarding the use and efficacy of ATP therapies in CHD patients. We describe our experience with ATPM in a diverse group of repaired CHD patients to determine utility and success of ATPM in patients with CHD.

\section{Methods}

All patients with CHD who underwent ATPM placement between 2000 and 2010 at a single institution were identified by query of the clinical cardiac device database. The objective was to define the use and efficacy of ATP in patients with CHD. The Investigational Review Board approved this study. A retrospective review of medical records was performed to identify the patients' congenital heart malformation and surgical history, history of IART, successful terminations of IART by the implanted device or by direct current cardioversion (DC) cardioversion required after ATPM implantation, and any device complications.

Device data, including AT detection, therapy data, and complications, were obtained from device diagnostics or other medical records. Successful ATP was defined as successful termination of IART by any pacing train in the ATP series. If the initial pacing train did not terminate tachycardia, but additional pacing therapies within the series did terminate tachycardia, the ATP series was considered to be successful. ATP was considered a failure if no pacing train within the ATP series successfully terminated tachycardia. Complications of device implant were evaluated. Early complications were defined as those occurring within 30 days of implant; late complications were those occurring thereafter.

As many patients were referred to our institution for device placement but primarily followed by cardiologists elsewhere, we included only those patients with follow-up data of more than 3 months in the final analysis.

\section{Results}

Between 2000 and 2010, 80 implants were performed in 72 patients (Table 1). The median age at first implant was 24.9 years (range 0.5 to 59.6 years).

Bradycardia implant indication was SND in 58 (81\%), CHB in 7 (10\%), and both SND and CHB in $2(3 \%)$. There were five patients $(7 \%)$ who had
Table 1. Patient Implant Characteristics

\begin{tabular}{lc}
\hline & $\mathrm{n}=72$ \\
\hline Age at ATPM implant, median (range) & 24.9 years $(0.5-59.6)$ \\
Gender & $44(61 \%)$ \\
Male & $50(70 \%)$ \\
Atrial tachycardia prior to implant & \\
Congenital heart disease & $25(35 \%)$ \\
Single ventricle & $25(35 \%)$ \\
Atrial switch procedure & $22(30 \%)$ \\
Other & \\
Implant indication & $58(81 \%)$ \\
Sinus node disease & $7(10 \%)$ \\
Complete heart block & $2(3 \%)$ \\
SND and CHB & $5(7 \%)$ \\
None & $68(94 \%)$ \\
Dual chamber system & $23(32 \%)$ \\
Ablation/Maze prior to implant & $27(38 \%)$ \\
Antiarrhythmic therapy & $16(22 \%)$ \\
Rate control (BB, CCB) & $24(33 \%)$ \\
Rhythm control (Class I or III) & \\
System/leads & $29(42 \%)$ \\
Epicardial & $36(52 \%)$ \\
Endocardial & $4(6 \%)$ \\
Combination & $23(32 \%)$ \\
Cardioversion prior to implant & $6(8 \%)$ \\
Death &
\end{tabular}

ATPM, antitachycardia pacemaker; BB, beta-blocker; CCB, calcium channel blocker; CHB, complete heart block; SND, sinus node disease.

recurrent refractory IART and underwent epicardial pacemaker placement during an otherwise planned sternotomy with no other documented bradycardia implant indication.

\section{Initial Implant Data}

Single-ventricle patients and those with atrial switch procedures, including both Mustard and Senning operations, comprised the majority of the patient group: 25 (35\%) patients had undergone Fontan palliation; 25 (35\%) had undergone atrial switch procedures. Other two-ventricle repairs $(\mathrm{n}=22 ; 30 \%)$, comprised the remaining patients. Thirty percent of ATPM patients had a bradycardia pacing indication and no documented IART before implant, but were felt to be at risk for IART by implanting physician due to CHD and surgical history.

Most patients, 64 (94\%), had a dual chamber system. The generator was in all cases designed for dual chamber functionality, though in some patients only a functional atrial lead was present, an off-label use of the device. Epicardial systems were placed in 29 (42\%), endocardial systems in 36 $(52 \%)$, and 4 patients $(6 \%)$ had a combination of epicardial and endocardial leads.

Prior to ATPM implant, 27 (38\%) patients were on antiarrhythmic therapy. Rate control therapy with beta-blockade or calcium channel blocker 
Table 2. Antitachycardia Pacing Results

\begin{tabular}{lc}
\hline Follow-up $>3$ months & $\mathrm{n}=56$ \\
\hline Follow-up, median (range) & 2.8 years $(0.3-10.7)$ \\
AT & $41(73 \%)$ \\
Prior to ATPM implant & $35(63 \%)$ \\
Post-ATPM implant & $29(52 \%)$ \\
Antiarrhythmic therapy after implant & $20(36 \%)$ \\
Successful ATP & $(57 \%)$ \\
AT post-ATPM implant, $\mathrm{n}=35$ & 1.3 years $(0-6.5)$ \\
Time to first successful ATP, median & \\
$\quad$ (range) & $6(30 \%)$ \\
Number of successful ATP, $\mathrm{n}=20$ & $1(5 \%)$ \\
$<5$ & $13(65 \%)$ \\
$5-15$ & $8(14 \%)$ \\
$>15$ & \\
DC cardioversion required after ATPM & \\
$\quad$ implant & \\
\hline
\end{tabular}

AT, atrial tachycardia; ATP, antitachycardia pacing; ATPM, antitachycardia pacemaker.

was used in $16(22 \%)$ patients. Rhythm control with class I or III antiarrhythmic agents was used in $24(33 \%)$ patients. Ablation or maze procedure was performed in $23(32 \%)$ patients prior to ATPM implant, and 23 (32\%) required cardioversion prior to ATPM implant.

There were six deaths (8\%), one of which occurred within 1 month of implant; this patient was an infant with hypoplastic left heart syndrome who died while being cannulated for extracorporeal membrane oxygenation (ECMO) 24 days after implant. None of the deaths was related to device implant or ATP.

\section{Follow-up Antitachycardia Pacing Results}

Follow-up data of more than 3 months were available for 56 patients; median follow-up time was 2.8 years (Table 2). Twenty (36\%) patients received successful ATP. First successful ATP occurred a median 1.3 years postimplant. More than 15 successful ATP episodes were documented in $13(65 \%)$ of the patients who received successful ATP. For those patients with IART after implant, ATP was successful in 20 (57\%) CHD patients. DC cardioversion after implant was required in eight (14\%) patients with IART not responsive to ATP, in contrast to the pre-ATPM implant rate of $32 \%(P=.02)$. Four patients who required cardioversion post-ATPM implant had not had a cardioversion prior to ATPM implant.

Of the three patients with single-chamber ATPM included in the follow-up analysis period, one had AT post-ATPM implantation, for which ATP was unsuccessful and required cardioversion. The remaining two patients had no AT documented postimplant.
Table 3. Clinical Factors Associated with Successful Antitachycardia Pacing

\begin{tabular}{lcc}
\hline Follow-up $>3$ months & $\mathrm{n}=56$ & $P$ \\
\hline Successful ATP, $\mathrm{n}=20$ & & .04 \\
Two-ventricle repair, $\mathrm{n}=38$ & $17(45 \%)$ & \\
One-ventricle palliation, $\mathrm{n}=18$ & $3(17 \%)$ & \\
\hline Atrial tachycardia prior to implant, $\mathrm{n}=41$ & $19(46 \%)$ & .006 \\
No atrial tachycardia prior to implant, $\mathrm{n}=15$ & $1(7 \%)$ & \\
\hline Atrial switch procedure, $\mathrm{n}=21$ & $9(43 \%)$ & $\mathrm{NS}$ \\
Other congenital heart surgery, $\mathrm{n}=35$ & $11(31 \%)$ & \\
\hline Preimplant AT, successful ATP, $\mathrm{n}=19$ & & .01 \\
Atrial switch procedure, $\mathrm{n}=12$ & $8(67 \%)$ & \\
Other congenital heart surgery, $\mathrm{n}=7$ & $2(28 \%)$ & \\
\hline
\end{tabular}

AT, atrial tachycardia; ATP, antitachycardia pacing.

Patients with two-ventricle repairs were more likely to have successful ATP than those with oneventricle palliation $(45 \%$ vs. $17 \%, P=.04)$ (Table 3). Patients with documented IART had more successful ATP than those with no documented IART prior to implant $(46 \%$ vs. $7 \%, P=$ .006). Overall, patients who had undergone atrial switch procedure had no difference in successful ATP compared with those after other surgeries (43\% vs. $31 \%, P=.39$ ). Of those patients with documented IART prior to ATPM implant, however, atrial switch patients were more likely to have successful ATP $(67 \%$ vs. $28 \%, P=.01)$.

\section{Complications}

Early complications of ATPM implant occurred in six patients (11\%), including lead failure, lead dislodgement, and excessive redundant lead requiring revision. Late complications after implant occurred in three patients (5\%), including infection requiring explant, pocket disruption secondary to trauma, and atrial lead failure. ATP was disabled due to acceleration of the ventricular rate or $1: 1$ conduction during ATP to prevent induction of a ventricular arrhythmia in six patients $(11 \%)$.

\section{Discussion}

IART is a major source of morbidity in patients with repaired or palliated CHD, frequently requiring combination therapy, which may include antiarrhythmic medications, ablation procedures, and long-term pacing. ${ }^{7-9}$ This study reports the utility and success of ATP in CHD patients. In our single-center cohort, ATP was successful in the majority of CHD patients with IART after implant. Those patients with atrial switch procedures and documented IART prior to implant had 
the highest incidence of successful ATP therapies. This study demonstrated a reduction in the need for cardioversion postimplant. It is important, however, to note that many patients underwent concomitant maze procedure/ablation and may have had medication changes, so direct benefit from ATP therapy could not be quantified. The fact that four patients who had not required cardioversion before ATPM implant later required cardioversion attests to both the evolution of IART in this population and likely the increased cardiac surveillance offered by a pacemaker.

The majority of complications related to device implant were related to lead failure or lead revision; these data are not significantly different from previously reported incidence of lead revision in CHD patients. ${ }^{10,11}$

In a large multicenter prospective study of patients with anatomically normal hearts and atrial tachyarrhythmias (AT) including atrial fibrillation, atrial flutter, and AT, implanted with AT500 and treated with antiarrhythmic medications, 30\% had no recurrence of AT in the follow-up period, suggesting that atrial pacing alone may serve to decrease AT. ${ }^{12}$ It is reasonable to conclude that CHD patients with SND and IART might also benefit from atrial pacing; in many of them, however, their venous connections and cardiac anatomy make this a technically challenging option. In this study, patients without documented IART prior to implant were unlikely to receive successful therapy from ATP. It seems logical that ATPM should not be implanted in patients without documented IART; however, this study did not evaluate other potential influences on ATP success. As implants in CHD patients can be complex, such as in those with Fontan palliation or atrial switch procedures, it remains important to consider the future possibility of IART at an implant principally for bradycardia. ATPM may still be a reasonable option at implant if the patient will not be limited by the few programming constraints of the ATP device. One important limitation is that pacemaker leads are not able to be programmed in the unipolar mode. Thus, the identification of those patients most likely to benefit from ATP remains the most effective strategy. The devices described in this study population have largely been supplanted by newer models, though the ATP programming options are similar. There remains a cost difference and level of programming complexity between ATPM and other bradycardia pacing devices, and it is important to select the appropriate device for the individual patient.
ATP programming per se has not been studied in the CHD population. In a recent analysis of successful ATP in adults without CHD, ramp pacing was determined to be more successful than double-extrastimulus ("Burst +") pacing ${ }^{13}$ and may be considered for further studies in this population to improve ATP. Additionally, it has been reported that in patients with tachy-brady syndrome without CHD, initial ramp ATP was successful in most patients who had successful ATP. ${ }^{14}$ This series represents no uniform strategy for atrial ATP programming, which should be further investigated in CHD patients.

\section{Conclusion}

ATP is an effective component in the multifaceted management of IART in postsurgical CHD, and spared numerous DC cardioversions in this series. Patients without documented IART prior to implant, however, were unlikely to receive successful therapy from ATPM systems. ATP therapies were most successful in patients with two-ventricle repairs, specifically atrial switch procedures with documented IART prior to implant.

\section{Author Contributions}

Anna N. Kamp, MD, participated in research concept/ design, data collection, statistics, data analysis/ interpretations, drafting the article, and approval of the article for publication.

Martin J. LaPage, MD, participated in interpretation of data, critical revision of the article, and approval of the article for publication.

Gerald A. Serwer, MD, participated in research concept/ design, data interpretations, critical revision of the article, and approval of the article for publication.

Macdonald Dick, II, MD, participated in research concept/ design, data interpretations, critical revision of the article, and approval of the article for publication.

David J. Bradley, MD, participated in research concept/ design, data collection, statistics, data analysis/ interpretations, critical revision of the article, and approval of the article for publication.

\section{Acknowledgement}

The authors would like to acknowledge the expert research assistance of Ms.Brynn Dechert-Crooks, PNP.

Corresponding Author: Anna N. Kamp, MD, MPH, Cardiology, The Heart Center, Nationwide Children's 
Hospital, 700 Children's Drive, Columbus, OH 43205 2664, USA. Tel: 614-722-0494; Fax: 614-722-2549; E-mail: anna.kamp@nationwidechildrens.org

Conflict of interest: Gerald A. Serwer received consulting fees from Medtronic. David J. Bradley has received a fellow training grant from Medtronic. Anna N. Kamp, Macdonald Dick, II, and Martin J. LaPage have no disclosures.

Accepted in final form: September 23, 2014.

\section{References}

1 Walsh EP, Cecchin F. Arrhythmias in adult patients with congenital heart disease. Circulation. 2007;115: 534-545.

2 Weipert J, Noebauer C, Schreiber C, et al. Occurrence and management of atrial arrhythmia after long-term Fontan circulation. 7 Thorac Cardiovasc Surg. 2004;127:457-464.

3 Kannankeril PJ, Fish FA. Management of intraatrial reentrant tachycardia. Curr Opin Cardiol. 2005;20:89-93.

4 Tsao S, Deal BJ, Backer CL, Ward K, Franklin WH, Mavroudis C. Device management of arrhythmias after Fontan conversion. 7 Thorac Cardiovasc Surg. 2009;138:937-940.

5 Stephenson E, Casavant D, Tuzi J, et al. Efficacy of atrial antitachycardia pacing using the Medtronic AT500 pacemaker in patients with congenital heart disease. Am 7 Cardiol. 2003;92:871-876.

6 Drago F, Silvetti MS, Grutter G, De Santis A. Long term management of atrial arrhythmias in young patients with sick sinus syndrome undergoing early operation to correct congenital heart disease. Europace. 2006;8:488-494.

7 Epstein AE, DiMarco JP, Ellenbogen KA, et al. ACC/AHA/HRS 2008 guidelines for device-based therapy of cardiac rhythm abnormalities: a report of the American College of Cardiology/American Heart Association Task Force on practice guidelines (writing committee to revise the ACC/AHA/ NASPE 2002 guideline update for implantation of cardiac pacemakers and antiarrhythmia devices) developed in collaboration with the American Association for Thoracic Surgery and Society of Thoracic Surgeons. 7 Am Coll Cardiol. 2008;51:e1-e62.

8 Villain E. Indications for pacing in patients with congenital heart disease. Pacing Clin Electrophysiol. 2008;31(suppl 1):S17-S20.

9 McLeod CJ, Asirvatham SJ, Warnes CA, Ammash NM. Device therapy for arrhythmia management in adults with congenital heart disease. Expert Rev Med Devices. 2010;7:519-527.

10 Fortescue E, Berul C, Cecchin F, Walsh E, Triedman J, Aexander M. Patient, procedural, and hardware factos associated with pacemaker lead failures in pediatrics and congenital heart disease. Heart Rhythm. 2004;1:150-159.

11 Smerup M, Hjertholm T, Johnsen S, et al. Pacemaker implantation after congenital heart surgery: risk and prognosis in a population-based follow-up study. Eur 7 Cardiothorac Surg. 2005;28:61-68.

12 Ricci R, Santini M, Padeletti L, et al. Atrial tachyarrhythmia recurrence temporal patterns in bradycardia patients implanted with antitachycardia pacemakers. 7 Cardiovasc Electrophysiol. 2004;15:4451.

13 Gulizia M, Mangiameli S, Orazi S, et al. Randomized comparison between Ramp and Burst+ atrial antitachycardia pacing therapies in patients suffering from sinus node disease and atrial fibrillation and implanted with a DDDRP device. Europace. 2006;8:465-473.

14 Schuster P, Faerestrand S, Ohm OJ. Device treatment of atrial tachycardia-minor additional effect of repeating pacing sequences. Int 7 Cardiol. 2005;104:10-14. 